PROCEEDINGS OF THE

AMERICAN MATHEMATICAL SOCIETY

Volume 128, Number 7, Pages 1971-1974

S 0002-9939(99)05336-8

Article electronically published on November 24, 1999

\title{
ON ORDER CONTINUOUS NORMS
}

\author{
SI-KIT CHUNG AND DENNY LEUNG
}

(Communicated by Dale Alspach)

\begin{abstract}
It is shown that a normed vector lattice $(E,\|\cdot\|)$ is order continuous if and only if, for every lattice norm $\rho$ on $E$ with $\rho \leq\|\cdot\|$, the $\|\cdot\|$-topology and $\rho$-topology coincide on every order interval of $E$.
\end{abstract}

\section{INTRODUCTION AND PRELIMINARIES}

A normed vector lattice $E$ is said to be order continuous, respectively, $\sigma$-order continuous, if $\lim _{\alpha}\left\|x_{\alpha}\right\|=0$ for every downward directed system, respectively, decreasing sequence, $\left(x_{\alpha}\right)_{\alpha \in \Lambda}$ in $E$ such that $\bigwedge_{\alpha \in \Lambda} x_{\alpha}=0$. It is said to satisfy the norm-Cauchy condition if every order bounded increasing sequence in $E$ is Cauchy. It is well-known that a normed vector lattice is order continuous if and only if it is $\sigma$-order continuous and satisfies the norm-Cauchy condition (see, e.g., 6, Theorem 103.4]). It is easy to observe that both $\sigma$-order continuity of the norm and the norm-Cauchy condition pass from a normed vector lattice to its norm completion. Hence, the norm completion of an order continuous normed vector lattice is also order continuous. Banach lattices, or more generally, normed vector lattices, with order continuous norms have been studied extensively and various characterizations of order continuity have been obtained (see [1, 5, 6]). The starting point of the present paper is the following result due to Amemiya (cf. [4, Theorem 2.4.8]).

Theorem 1.1. Let $\|\cdot\|_{1}$ and $\|\cdot\|_{2}$ be two order continuous norms on a vector lattice $E$, and suppose that $\left(E,\|\cdot\|_{i}\right)$ is an ideal in its norm completion for $i=1,2$. Then on every order interval of $E$, the norm topologies coincide and the weak topologies coincide.

We will show that the theorem holds without the assumption that $\left(E,\|\cdot\|_{i}\right)$ is an ideal in its norm completion. In fact, the conclusion of Amemiya's Theorem gives a characterization of the order continuity of the norms involved (see Theorem 2.3). We begin with some examples which show that the conclusion of Amemiya's Theorem no longer holds under certain weakenings of the hypothesis.

Example 1.1. Let $E$ be the vector lattice $C[0,1]$. Let $\|\cdot\|_{1}$ be the $L^{1}$-norm, and let $\|\cdot\|_{2}$ be given by $\|f\|_{2}=\int_{A}|f|$, where $A$ is a measurable subset of $[0,1]$ with measure less than 1 , but the measure of the intersection of $A$ with any open interval contained in $[0,1]$ is positive. Clearly, both $\|\cdot\|_{1}$ and $\|\cdot\|_{2}$ are lattice norms on

Received by the editors May 4, 1998 and, in revised form, August 13, 1998.

1991 Mathematics Subject Classification. Primary 46E30.

Key words and phrases. Normed vector lattices, order continuous norms.

(C)2000 American Mathematical Society 
$E$ which satisfy the norm-Cauchy condition. However, the topologies generated by the two norms differ on the order interval $[0, \mathbf{1}]$, where $\mathbf{1}$ denotes the constant 1 function.

Example 1.2. Let $E$ be the vector lattice of all functions in $L^{1}[0,1]$ which is (equal almost everywhere to a function that is) continuous at 0 . If $f \in E$, let $\rho(f)=|g(0)|$, where $g=f$ almost everywhere, and $g$ is continuous at 0 . It is clear that $\rho$ is a welldefined lattice seminorm on $E$. Let $\|\cdot\|_{1}$ be the $L^{1}$-norm, and let $\|\cdot\|_{2}=\|\cdot\|_{1}+\rho$. Then $\|\cdot\|_{1}$ is an order continuous norm on $E$, and $\|\cdot\|_{2}$ is a lattice norm on $E$ which satisfies the norm-Cauchy condition. However, the topologies generated by $\|\cdot\|_{1}$ and $\|\cdot\|_{2}$ differ on $[0, \mathbf{1}]$, where $\mathbf{1}$ is the constant 1 function.

Example 1.3. Let $E$ be a vector lattice, and suppose $\|\cdot\|_{1}$ is a $\sigma$-order continuous norm on $E$ which is not order continuous. By Theorem 2.3, there exists a lattice norm $\|\cdot\|_{2}$ on $E$ such that $\|\cdot\|_{2} \leq\|\cdot\|_{1}$, and the topologies generated by the two norms differ on some order interval of $E$. Thus, the conclusion of Amemiya's Theorem may still fail to hold even if both norms are assumed to be $\sigma$-order continuous.

\section{MAIN RESULTS}

In this section, we give the proof of the generalization of Amemiya's Theorem mentioned above, as well as certain characterizations of order continuity in normed vector lattices. The way in which order continuity is applied in this circle of ideas is contained in the next proposition.

Proposition 2.1. Let $\|\cdot\|_{1}$ and $\|\cdot\|_{2}$ be two lattice norms on a vector lattice $E$. For $\alpha=1,2$, let $\widehat{E_{\alpha}}$ be the norm completion of $E_{\alpha}=\left(E,\|\cdot\|_{\alpha}\right)$. Suppose the identity map $i: E_{1} \longrightarrow E_{2}$ is continuous and $E_{1}$ is order continuous. Then the extension $\hat{i}: \widehat{E_{1}} \longrightarrow \widehat{E_{2}}$ of $i$ is one-to-one.

Proof. Let $F$ be the $\sigma\left(E_{1}^{\prime}, \widehat{E_{1}}\right)$-closure of $i^{\prime}\left(E_{2}^{\prime}\right)$. Then $F$ is a band in $E_{1}^{\prime}$. Since $E_{1}$ is order continuous, by [6, Corollary 106.4], $F$ is $\sigma\left(E_{1}^{\prime}, E_{1}\right)$-closed. As $i$ is one-to-one, $i^{\prime}\left(E_{2}^{\prime}\right)$ is $\sigma\left(E_{1}^{\prime}, E_{1}\right)$-dense in $E_{1}^{\prime}$. Therefore, $F=E_{1}^{\prime}$. Hence $i^{\prime}\left(E_{2}^{\prime}\right)$ is $\sigma\left(E_{1}^{\prime}, \widehat{E_{1}}\right)$-dense in $E_{1}^{\prime}$. It follows that $\hat{i}$ is one-to-one.

We are now ready to prove the promised generalization of Amemiya's Theorem.

Theorem 2.2. Let $E$ be a vector lattice. Suppose $\|\cdot\|_{1}$, respectively $\|\cdot\|_{2}$, is an order continuous norm, respectively, $\sigma$-order continuous norm, on $E$. Then on every order interval of $E$, the $\|\cdot\|_{1}$-topology and $\|\cdot\|_{2}$-topology coincide and the $\sigma\left(E,\left(E,\|\cdot\|_{1}\right)^{\prime}\right)$-topology and $\sigma\left(E,\left(E,\|\cdot\|_{2}\right)^{\prime}\right)$-topology coincide.

Proof. First, we show that $\|\cdot\|_{2}$ is in fact order continuous. Let $\left(x_{\alpha}\right)_{\alpha \in \Lambda}$ be a decreasing net in $E$ with $\inf _{\alpha \in \Lambda} x_{\alpha}=0$. Fix $\alpha_{0} \in \Lambda$. Let $E_{\alpha_{0}}$ be the principal ideal of $E$ generated by $x_{\alpha_{0}}$. Since $\left(E,\|\cdot\|_{1}\right)$ is order continuous, by 6 . Theorem 103.12], there exists $x^{\prime} \in\left(E,\|\cdot\|_{1}\right)_{+}^{\prime}$ such that $x^{\prime}$ is strictly positive on $E_{\alpha_{0}}$. Since $\lim _{\alpha \in \Lambda}\left\|x_{\alpha}\right\|_{1}=0, \lim _{\alpha \in \Lambda}\left\langle x_{\alpha}, x^{\prime}\right\rangle=0$. Hence there exist $\alpha_{n} \in \Lambda, n \in \mathbf{N}$, with $\alpha_{n+1} \leq \alpha_{n}$ for all $n \in \mathbf{N}$ such that $\lim _{n \rightarrow \infty}\left\langle x_{\alpha_{n}}, x^{\prime}\right\rangle=0$. Since $x^{\prime}$ is strictly positive on $E_{\alpha_{0}}$, we have $\inf _{n \in \mathbf{N}} x_{\alpha_{n}}=0$. The $\sigma$-order continuity of $\|\cdot\|_{2}$ implies that $\inf _{n \in \mathbf{N}}\left\|x_{\alpha_{n}}\right\|_{2}=0$ and so $\inf _{\alpha \in \Lambda}\left\|x_{\alpha}\right\|_{2}=0$.

Next we prove the required result for the case where $\left(E,\|\cdot\|_{1}\right)$ is a Banach lattice and $\|\cdot\|_{1} \geq\|\cdot\|_{2}$. Denote the weak topologies $\sigma\left(E,\left(E,\|\cdot\|_{1}\right)^{\prime}\right)$ and $\sigma\left(E,\left(E,\|\cdot\|_{2}\right)^{\prime}\right)$ by $\sigma_{1}$ and $\sigma_{2}$ respectively. Let $x \in E_{+}$. Since $\left(E,\|\cdot\|_{1}\right)$ is an order continuous 
Banach lattice, the order interval $[0, x]$ is $\sigma_{1}$-compact. Note that the identity map $i:\left([0, x], \sigma_{1}\right) \rightarrow\left([0, x], \sigma_{2}\right)$ is continuous and that $\sigma_{2}$ is Hausdorff, so $\sigma_{1}=\sigma_{2}$ on $[0, x]$. Suppose $\left(x_{n}\right)$ is a sequence in $[0, x]$ with $\lim _{n \rightarrow \infty}\left\|x_{n}\right\|_{2}=0$. Then $x_{n} \stackrel{\sigma_{2}}{\rightarrow} 0$ and so $x_{n} \stackrel{\sigma_{1}}{\rightarrow} 0$. By [4. Proposition 2.3.4], there exists a disjoint sequence $\left(x_{n}^{\prime}\right)$ in the positive part of the unit ball of $\left(E,\|\cdot\|_{1}\right)^{\prime}$ such that $\limsup _{n \rightarrow \infty}\left\langle x_{n}, x_{n}^{\prime}\right\rangle=$ $\lim \sup _{n \rightarrow \infty}\left\|x_{n}\right\|_{1}$. By [4, Corollary 2.4.3], we have $\lim _{n \rightarrow \infty}\left\langle x, x_{n}^{\prime}\right\rangle=0$. Therefore $\lim _{n \rightarrow \infty}\left\langle x_{n}, x_{n}^{\prime}\right\rangle=0$ and so $\lim _{n \rightarrow \infty}\left\|x_{n}\right\|_{1}=0$.

For the general case, we let $\rho=\|\cdot\|_{1}+\|\cdot\|_{2}$. It suffices to prove the required result for $\rho$ and $\|\cdot\|_{1}$. Let $E_{0}$ and $E_{1}$ be the norm completions of $(E, \rho)$ and $\left(E,\|\cdot\|_{1}\right)$ respectively (with the norms still denoted by $\rho$ and $\|\cdot\|_{1}$ respectively). By Proposition [2.1, the extension $\hat{i}:\left(E_{0}, \rho\right) \rightarrow\left(E_{1},\|\cdot\|_{1}\right)$ of the identity map $i:(E, \rho) \rightarrow\left(E,\|\cdot\|_{1}\right)$ is one-to-one. We may identify $E_{0}$ and $\hat{i}\left(E_{0}\right)$ and so we have two lattice norms $\rho$ and $\|\cdot\|_{1}$ on $E_{0}$. Since $E_{0}$ is the norm completion of the order continuous normed vector lattice $(E, \rho),\left(E_{0}, \rho\right)$ is order continuous. The required result then follows from the result on the special case above.

Theorem 2.3. Let $(E,\|\cdot\|)$ be a normed vector lattice, and let $\widehat{E}$ be its norm completion. The following assertions are equivalent.

(1) $(E,\|\cdot\|)$ is order continuous.

(2) For every lattice norm $\rho$ on $E$ with $\rho \leq\|\cdot\|$, the $\|\cdot\|$-topology and $\rho$-topology coincide on every order interval of $E$.

(3) $E$ is strongly order dense in $\widehat{E}$, and $\widehat{E}$ is order continuous.

Proof. (3) $\Longrightarrow(1)$ Obvious.

$(1) \Longrightarrow(2)$ This follows easily from Theorem 2.2

$(2) \Longrightarrow(3)$ We divide the proof into two parts.

(I) Suppose $(\widehat{E},\|\cdot\|)$ is not order continuous. Then by [3, Satz 8$]$, there exists a disjoint sequence $\left(x_{n}\right)$ in $\widehat{E}$ contained in an order interval $[0, y]$ of $\widehat{E}$ such that $\left\|x_{n}\right\|=1$ for all $n \in \mathbf{N}$. We may assume that $y \in E$. Let $P_{n}$ (respectively $P$ ) be the band projection from $\widehat{E}^{\prime \prime}$ onto the band generated in $\widehat{E}^{\prime \prime}$ by $x_{n}$ (respectively $\left.\left\{x_{n}: n \in \mathbf{N}\right\}\right)$. Define a lattice norm $\rho$ on $\widehat{E}$ by $\rho(x)=\frac{1}{2}\left(\|x-P x\|+\sum_{n=1}^{\infty} \frac{\left\|P_{n} x\right\|}{2^{n}}\right)$. Then $\rho \leq\|\cdot\|$ on $\widehat{E}$ and $\rho\left(x_{n}\right)=\frac{1}{2^{n+1}}$. Choose a sequence $\left(y_{n}\right)$ in $E$ such that $\lim _{n \rightarrow \infty}\left\|y_{n}-x_{n}\right\|=0$. We may assume $0 \leq y_{n} \leq y$. Since $\rho\left(y_{n}\right) \leq \rho\left(y_{n}-x_{n}\right)$ $+\rho\left(x_{n}\right) \leq\left\|y_{n}-x_{n}\right\|+\rho\left(x_{n}\right) \rightarrow 0$ as $n \rightarrow \infty$, it follows from the assumption on $E$ that $\lim _{n \rightarrow \infty}\left\|y_{n}\right\|=0$. Hence we have $\lim _{n \rightarrow \infty}\left\|x_{n}\right\|=0$, a contradiction.

(II) Suppose $E$ is not strongly order dense in $\widehat{E}$. Let $0<x \in \widehat{E}$ be such that $[0, x] \cap E=\{0\}$. We may assume that $x<y$ for some $y \in E$. Let $B$ be the band in $\widehat{E}$ generated by $y$ and $B^{\perp}$ be its disjoint complement. Since $(\widehat{E},\|\cdot\|)$ is order continuous, by [2, Theorem 1.b.14], $B$ may be represented as an ideal of $L^{1}(\Omega, \Sigma, \mu)$ for some probability space $(\Omega, \Sigma, \mu)$ so that $y$ corresponds to the constant 1 function and $B^{\prime}$ consists of all measurable functions $g$ such that $\int_{\Omega}|g h| d \mu<\infty$ for all $h \in B$.

Fix $\epsilon>0$. Let $A=\{\omega \in \Omega: x(\omega)<\epsilon\}$. Note that $\chi_{A}$, that is, the map given by $h \mapsto \int_{A} h d \mu$ for $h \in B$, belongs to $(B,\|\cdot\|)^{\prime}$. Define a lattice semi-norm $\rho_{A}$ on $\widehat{E}$ by $\rho_{A}(h+z)=\max \left\{\left(\left\|\chi_{A}\right\|_{(B,\|\cdot\|)^{\prime}}+1\right)^{-1} \int_{A}|h| d \mu,\|z\|\right\}$ for $h \in B, z \in B^{\perp}$. It is clear that $\rho_{A} \leq\|\cdot\|$ on $\widehat{E}$. Moreover, $\rho_{A}$ is a lattice norm on $E$. Indeed, if $h+z \in E$ where $h \in B, z \in B^{\perp}$ and $\rho_{A}(h+z)=0$, then $z=0$ and $\int_{A}|h| d \mu=0$. So $h=0$ a.e. on $A$. Hence $\epsilon(|h| \wedge y) \in[0, x] \cap E$. Therefore $|h| \wedge y=0$ and so $h=0$. 
Since $0 \leq \chi_{\Omega \backslash A} \in B$, a sequence $\left(y_{n}\right)$ exists in $E_{+}$such that

$$
\lim _{n \rightarrow \infty}\left\|y_{n}-\chi_{\Omega \backslash A}\right\|=0 .
$$

Let $h_{n}=y_{n} \wedge y$. Then $\lim _{n \rightarrow \infty}\left\|h_{n}-\chi_{\Omega \backslash A}\right\|=0$. So $\rho_{A}\left(h_{n}\right)=\int_{A} h_{n} d \mu=$ $\int_{A}\left(h_{n}-\chi_{\Omega \backslash A}\right) d \mu \leq\left\|\chi_{A}\right\|_{(B,\|\cdot\|)^{\prime}}\left\|h_{n}-\chi_{\Omega \backslash A}\right\| \rightarrow 0$ as $n \rightarrow \infty$. Hence by the assumption on $E, \lim _{n \rightarrow \infty}\left\|h_{n}\right\|=0$ and so $\chi_{\Omega \backslash A}=0$. Thus

$$
\mu(\{\omega \in \Omega: \epsilon \leq x(\omega)\})=0 \text { for all } \epsilon>0
$$

and so we have $x=0$, a contradiction.

\section{REFERENCES}

[1] C. Aliprantis and O. Burkinshaw, Positive Operators, Academic Press, New York and London, 1985. MR 87h:47086

[2] J. Lindenstrauss and L. Tzafriri, Classical Banach Spaces II, Function Space, Springer-Verlag, Berlin, 1979. MR 81c:46001

[3] P. Meyer-Nieberg, Charakterisierung einiger topologischer und ordnungstheoretischer Eigenschaften von Banachverbänden mit Hilfe disjunkter Folgen, Arch. Math., 24 (1973), 640-647. MR 49:5771

[4] , Banach Lattices, Springer-Verlag, New York, 1991. MR 93f:46025

[5] H. H. Schaefer, Banach Lattices and Positive Operators, Springer-Verlag, Berlin, 1974. MR 54:11023

[6] A. C. Zaanen, Riesz Spaces II, North-Holland, 1983. MR 86b:46001

Department of Mathematics, University of Hong Kong, Hong Kong

E-mail address: schung@submaths.hku.hk

Department of Mathematics, National University of Singapore, Singapore 119260, SingAPORE

E-mail address: matlhh@nus.edu.sg 